\title{
Solid State Generator for Powerful Radio Frequency Ion Sources in Neutral Beam Injection Systems
}

\author{
W. Kraus, U. Fantz, B. Heinemann, P. Franzen, C. Martens \\ Max-Planck-Institut für Plasmaphysik, Boltzmannstr. 2, D-85748 Garching, Germany
}

\begin{abstract}
Radio frequency ion sources used in neutral beam injection systems (NBI) of fusion machines are currently supplied by self-excited RF generators. These generators have both a low power efficiency and a limited frequency stability, therefore transistorized amplifiers are being considered for the power supply of the next generation of RF sources. A $75 \mathrm{~kW}$ generator, originally designed for broadcasting, has been tested with a negative ion source. High operational reliability and a very good matching to the plasma load has been demonstrated. These results make this generator type a very promising candidate for future NBI systems.
\end{abstract}

\section{Introduction}

Powerful RF ion sources are operated at the Max-Planck-Institut für Plasmaphysik (IPP) on the ion source test facilities and in the neutral beam injection system of the fusion facility ASDEX-Upgrade [1, 2]. In the future, the fusion machines Wendelstein W7-X [3] and ITER will use RF ion sources for the generation of heating beams or diagnostic beams [4, 5]. To this time the RF sources have used self-excited RF generators with one or two tubes (triodes or tetrodes). An inherent drawback of these oscillators is the low electrical efficiency (less than $60 \%$ ). The ITER NBI each source will be supplied by four $200 \mathrm{~kW} / 1 \mathrm{MHz}$ generators, which require a primary power of approx. $1.4 \mathrm{MW}$ and an additional $600 \mathrm{~kW}$ water cooling system [4].

Another disadvantage of self-excited oscillators is difficult matching due to the unstable output frequency. At IPP ten different $1 \mathrm{MHz}$ self-excited generators with powers from 100 $\mathrm{kW}$ to $180 \mathrm{~kW}$ are in operation. Perfect matching on any generator has never been achieved. The impedance at the generator output is, in all cases, greater than $50 \Omega$ and the power factor $\cos \varphi$ is smaller than one. The reason is that the generator frequency is affected by the load and changes with the matching capacitance until the frequency flips and the matching breaks down.

In the future it is doubtful that the high power tubes needed for the RF circuit will be easily replaced. The impact of this both on source reliability and maintainability should not be underestimated.

The use of state-of-the-art solid state based generators can avoid these problems. Due to the much higher efficiency (about $90 \%$ ) the power consumption is considerably lower and 
water cooling is no longer necessary. As they have a fixed frequency matching is expected to be both easier and more stable.

Although these are striking arguments, however, the solid state generator needs to be experimentally validated by practical experience on plasma devices like those foreseen for NBI systems.

It is of particular importance to determine if the solid-state parts can stand operation with high reflected power and how the characteristics of the matching to the plasma load changes due to the generator having a fixed frequency. Experiments have been carried out at IPP on the negative ion source at the BATMAN test facility [1]. The previous power supply was a $100 \mathrm{~kW}$ self-excited generator with two triodes in a push-pull arrangement. A $75 \mathrm{~kW}$ solid state generator has been connected to the matching circuit of the ion source. As this circuit is unchanged it allowed direct comparison between the two kinds of generators. The commissioning and the first operational experience have been described in [6]. In this paper more detail on the matching and beam extraction experiments is provided.

\section{The solid state generator}

For the solid state generator a radio transmitter (SCIAMP 75, "scientific amplifier") of TRANSRADIO SenderSysteme Berlin AG has been chosen. This generator was originally designed as a $50 \mathrm{~kW}$ AM broadcast transmitter ("TRAM 50", [7]) and has been modified according to the requirements of an ion source operation at $1 \mathrm{MHz}$. The generator is aircooled and can run in steady-state operation.

For many years, solid state generators have replaced the tube generators in the field of radio broadcast. The advantages of using such well-designed series products for the power supply of RF ion sources are a potentially higher reliability and lower costs compared to the oscillators which are in end effect preproduction prototypes.

The tested solid state generator consists of 50 modules of maximum $1.5 \mathrm{~kW}$. In each module up to $260 \mathrm{~V}$ is switched by four transistors. The resulting square waves are arranged in parallel on a "combiner" ferrite core and then transformed into a sinusoidal wave by an output filter. Even if one or more modules should fail, the generator is still operable, although at a lower maximum output power. A module can be exchanged in a short time which increases the systems reliability and maintainability compared to that of self-excited generators. Powerful generators up to $2 \mathrm{MW}$ are available that consist of several of these 75 kW units. Contrary to tube based oscillators there is no high voltage inside the generator and, therefore, no stored energy that has to be removed during a fast shutdown. The modular concept and the lack of high voltage are other reasons to expect a high reliability.

For the solid state generator the forward and the reflected power are measured by directional couplers. This is in contrast to the oscillators used at IPP, where the RF power is calculated from $\cos \varphi$, RF voltage and current. This method, however, is no more state-ofthe-art. 
In order to allow source operation with a certain degree of mismatching, the maximal possible SWR (standing wave ratio) was adjusted to be 2 . This enables the generator to operate with a real load between $25 \Omega$ and $90 \Omega$. If the SWR exceeds 2 , corresponding to a reflected power of $11 \%$, the generator is protected by fast switch-off of the RF power. This becomes the self-protection for the case that the plasma does not ignite or vanishes during source operation. There are two exceptions: For the first $\mathrm{ms}$ of a pulse the self-protection is not activated in order to allow a high reflected power during the start-up of the plasma (see section 3.1) and below $20 \mathrm{~kW}$ a maximum SWR of slightly higher than 2 is tolerated.

In the oscillators, motor driven variable capacitors in the tank circuit have to be used to change the frequency [8]. This is a slow procedure and is only possible over a small range of about $20 \mathrm{kHz}$ due to the available change of capacitance. In the solid state generator a frequency variation can be performed electronically in $1 \mathrm{kHz}$ steps over a maximum range of $+/-50 \mathrm{kHz}$. In [6] it has been shown that the matching by frequency change is the equivalent to that made by changing the capacitance. The fast frequency change without mechanical components would it make possible to establish in a next step an automatic matching system.

The generator is connected to the $\mathrm{S} 7$ testbed controller unit via glass fiber lines and by a 25 $\mathrm{m}$ coaxial cable to the matching unit of the source as shown in Fig. 1. In order to keep the generator on ground potential during ion beam extraction the source is separated by a ferrite core insulation transformer with a transformation ratio of 1:3. In the used source at the BATMAN testbed the RF power is inductively coupled into a cylindrical volume of $25 \mathrm{~cm}$ diameter and a length of $12 \mathrm{~cm}$ ("driver"), out of which the plasma is flowing into the main chamber (width $x$ length $\times$ depth $=30 \times 59 \times 25 \mathrm{~cm}^{3}$ ). The alumina sidewalls of the driver are protected by an internal Faraday shield from plasma erosion and are surrounded by a coil of six windings. The source design and the matching unit are described in detail in [9]. The ITER source will have eight of these "drivers". The source is operated in Hydrogen or Deuterium in a duty cycle of $6 \mathrm{~s}$ pulse / 3 min interruption.

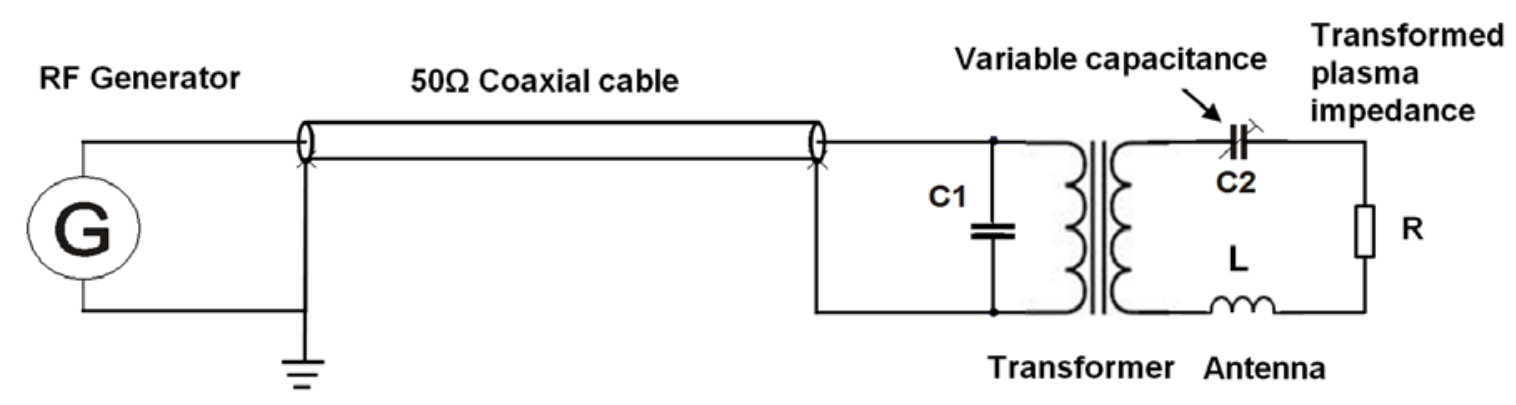

Figure 1: Matching circuit of the negative ion source 


\section{Experimental results}

\subsection{Starting the plasma with the solid state generator}

To ignite the discharge a small starter filament and a pulsed high pressure is necessary. The critical time for the matching is the initial phase of the plasma, as the plasma load is transient. Fig. 2 shows the voltage of the forward and reflected waves which are proportional to the square root of the forward and reflected power respectively during the

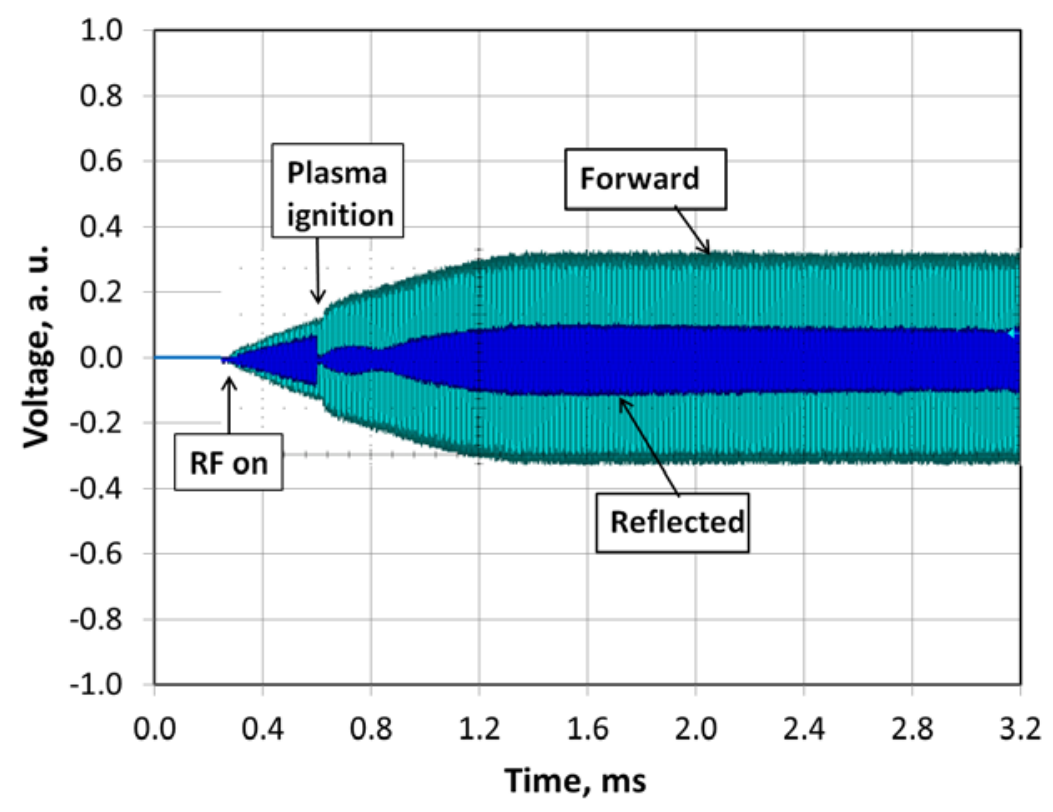

Figure 2: Time curves of the voltages of the forward and of the reflected wave during the plasma start-up phase

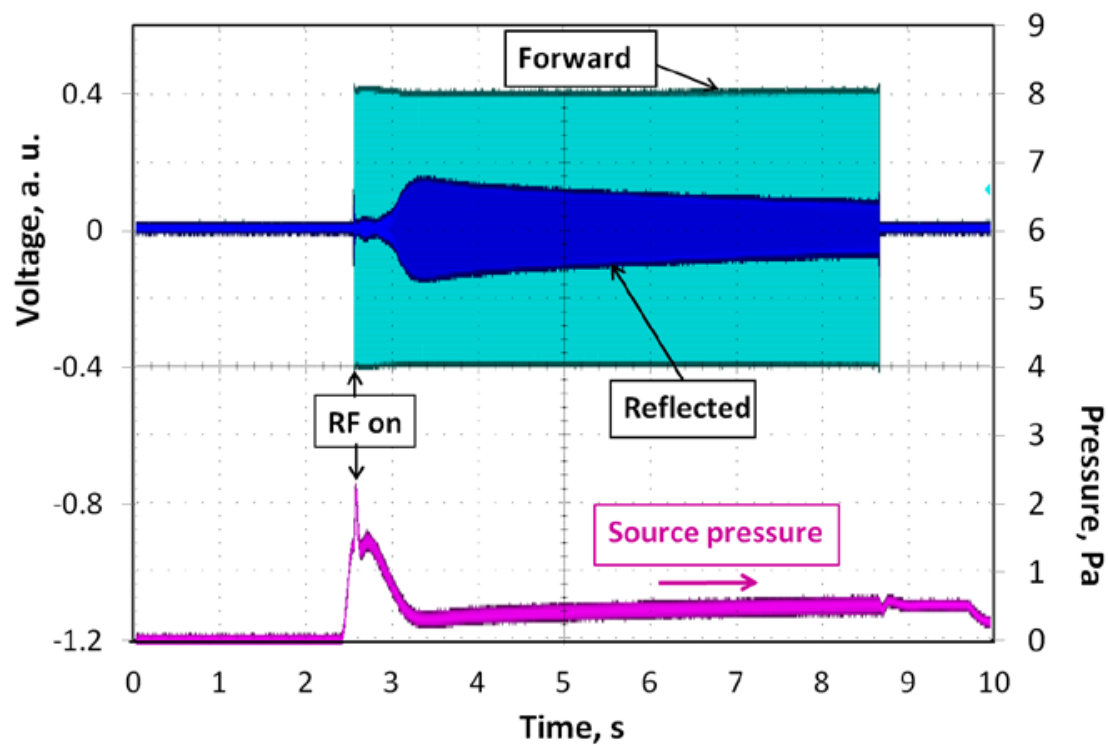

Figure 3: Time curves of the voltages of forward wave, reflected wave and source pressure during a 6 s plasma pulse

plasma start up. The reflected power in the first $0.3 \mathrm{~ms}$, before plasma ignition, is high. The reflected power drops immediately after the plasma has ignited. 
The pressure in the source varies between 1.5 Pa during the start pulse peak and the normal operation value of $0.4-0.6 \mathrm{~Pa}$. After the end of the pressure pulse the pressure increases slightly because of the decrease in the pumping speed of the testbed. The SWR is not constant during the pulse because of the reflected power is dependent on the source pressure (see Figure 3). It is still possible to match with SWR close to 1 , i. e. nearly no reflected power as shown in Figure 4. The SWR denoted in the following graphs is always the average value calculated for the second half of the pulse.

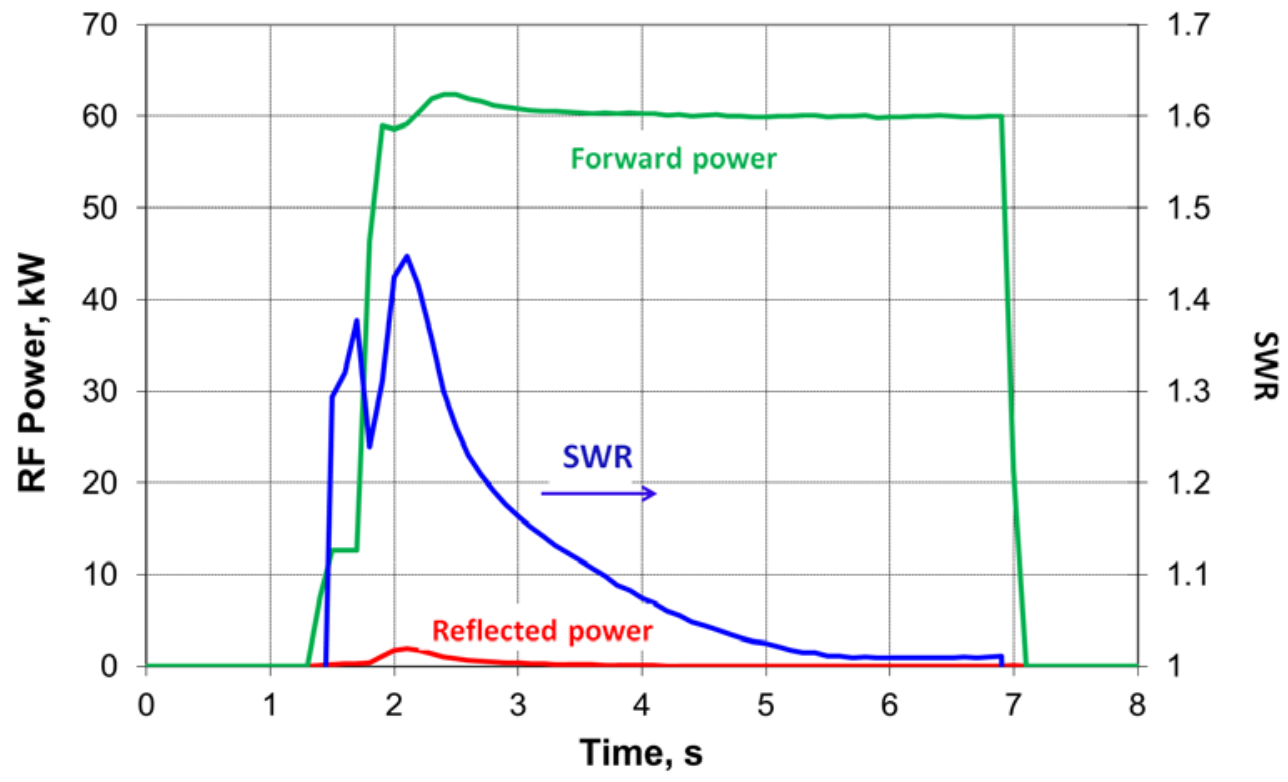

Figure 4: Example of a $60 \mathrm{~kW}$ pulse with SWR=1

Mismatching during the pressure pulse and protection of the generator during the start phase from too much reflected power has been avoided in two ways. First during the initial $500 \mathrm{~ms}$ the frequency was raised by $5 \mathrm{kHz}$ because for the same SWR a slightly higher frequency is required at high pressure (see section 3.2). In addition the power was reduced to $10-20 \mathrm{~kW}$, because the maximum allowed SWR is higher at low power. In this way the plasma can be started reliably under all conditions. A typical plasma pulse including the start phase is shown in Figure 4.

\subsection{Matching characteristics of oscillator and solid state generator}

With the solid state generator a variation by about $+/-15 \mathrm{kHz}$ is sufficient to cover the range of the allowed SWR values. This corresponds to a capacitance change of $0.1 \mathrm{nF}$, if the matching is done by tuning the series capacity $C 2$ [6]. This narrow range is mainly due to the high quality factor $(Q=\omega L / R)$ of the matching circuit, which is greater than 30 for an inductance $L$ of $10 \mu \mathrm{H}$ and a transformed plasma impedance $R$ of $2 \Omega$.

In Figure 5 it is shown for the BATMAN testbed source that the matching curve, as a function of the capacitance is broader for the previously used self-excited generator than for the amplifier. The reason for the broadening is the non-constant frequency. The frequency of the generator can vary over a range of up to $20 \mathrm{kHz}$. This variation is because changes of the 
inductance or capacitance of the matching circuit are transformed into the tank circuit of the generator and thus affect the generator frequency. Another reason allowing the self-excited generator to match over a broader range is that they tolerate a higher reflected power than the solid state generator.

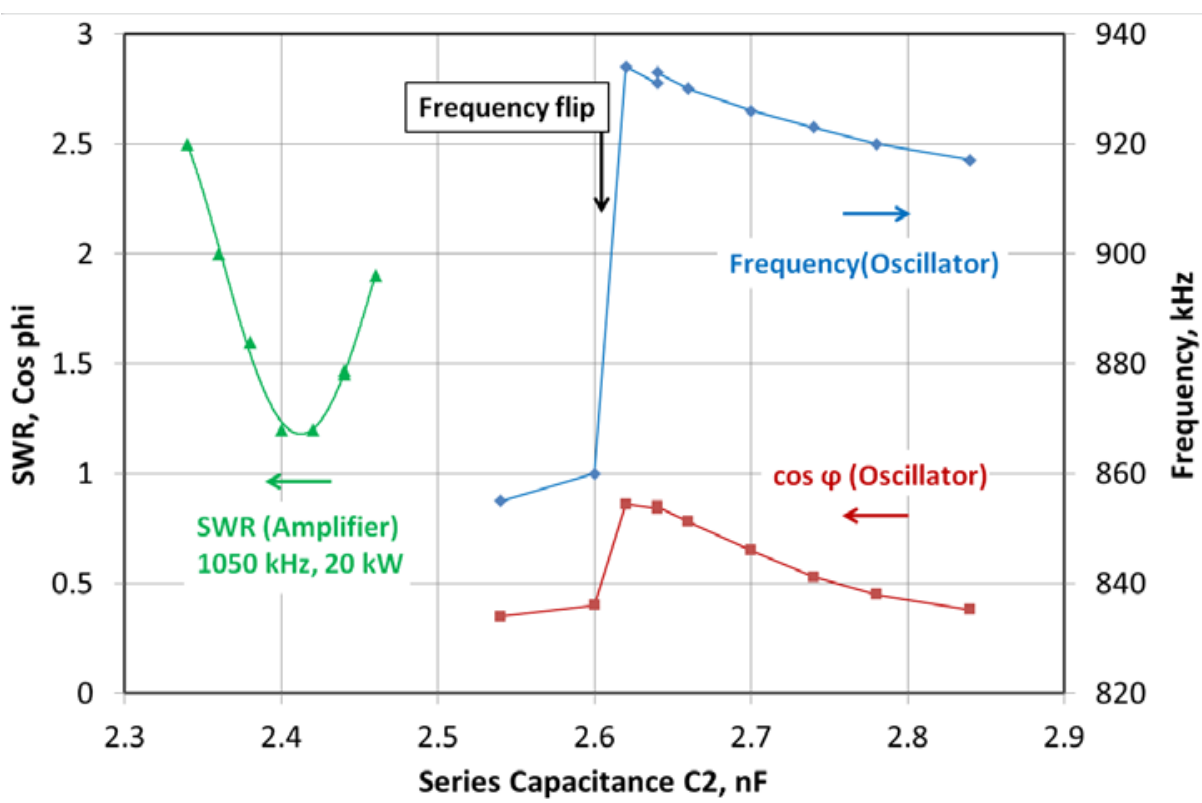

Figure 5: SWR of the amplifier and frequency and $\cos \varphi$ of the self-excited generator of the BATMAN testbed as a function of the series capacitance $C 2$

Common to all oscillators used at IPP is that perfect matching to the plasma source by changing the series capacity is not possible, because if the resistance at the generator output approaches $50 \Omega$ the frequency jumps by about $100 \mathrm{kHz}$ and more (see Fig. 5). At this "frequency flip" the matching breaks down and the generator is switched off. The reason for these frequency flips is not completely understood. It is probably caused by the mutual influence of the resonant circuits of the generator and the source. The low damping (or high Q) of the matching circuit contributes also to this effect. This frequency instability depends on the particular used generator. Influences are: the $Q$ of the tank circuit, the anode voltage and if the generator uses one tube or two tubes in push-pull arrangement. The inductance of the isolation transformer also plays an important role (see chapter 3.3). Examples of matching experiments with self-excited oscillators can be found in [10] and [11]. For safe operation without frequency jumps the matching has to be adjusted to a higher resistance and $\cos \varphi$ values which are somewhat below the achievable maximum. A typical setting is $\cos \varphi=0.8-0.9$ and $R=70 \Omega-90 \Omega$ [11]. This corresponds to a standing wave ratio (SWR) of 1.5 to 2 and a reflected power of $4 \%$ to $11 \%$.

\subsection{Matching with the solid state generator at different plasma parameters}

With the solid state generator it is possible to achieve a SWR below 1.2 corresponding to a reflected power of less than 1\% (see Figs 4, 6 and 7). It was, however, not clear, if the narrow matching range would be sufficient to cover the typical range of operational parameters. 
Figure 6 shows the matching for source operation at $0.4 \mathrm{~Pa}$ and $60 \mathrm{~kW}$. If the power is reduced to $20 \mathrm{~kW}$, the matching is essentially unaffected. The resistance and the inductance of the plasma do obviously not depend on the power or the plasma density. When the source pressure is raised from $0.4 \mathrm{~Pa}$ to $0.8 \mathrm{~Pa}$ the matching curve is shifted by $7 \mathrm{kHz}$ which corresponds to a slight increase of the inductance. However, the curves do overlap and it is possible to find a setting, for which the source can be run independent of pressure without needing to adjust the generator frequency.

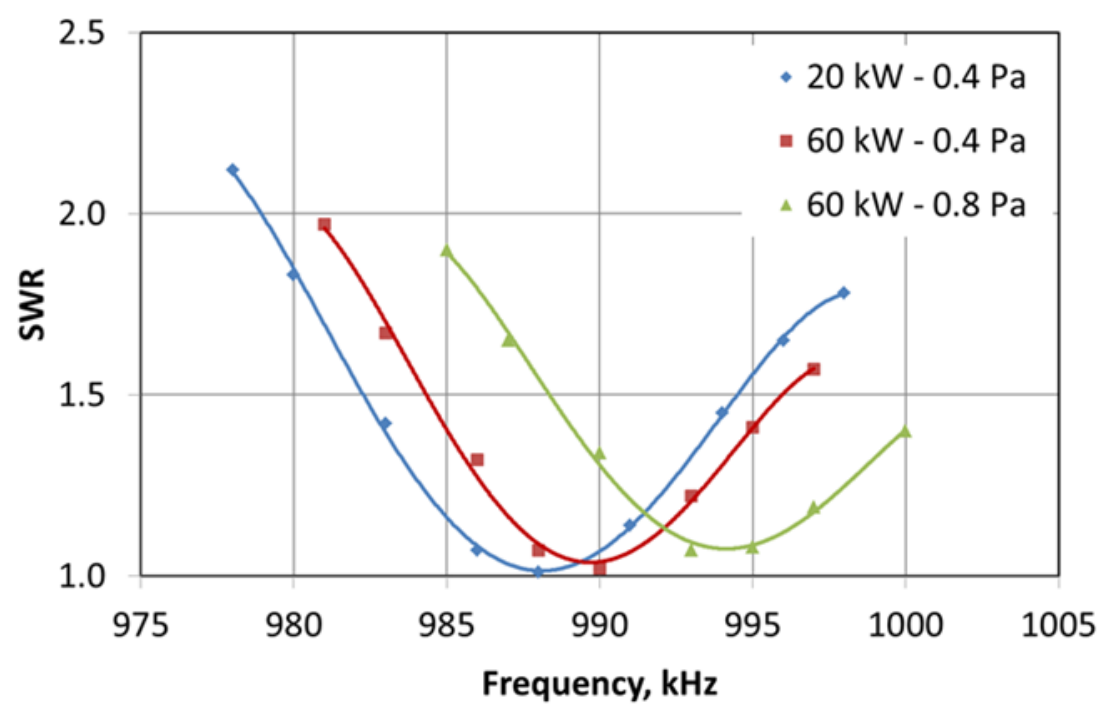

Figure 6: Change of the matching, when the power is reduced or the pressure is doubled.

The results are valid, if the working gas is changed from Hydrogen to Deuterium, although there is a shift of the whole matching curve by $10 \mathrm{kHz}$ (see Figure 7).

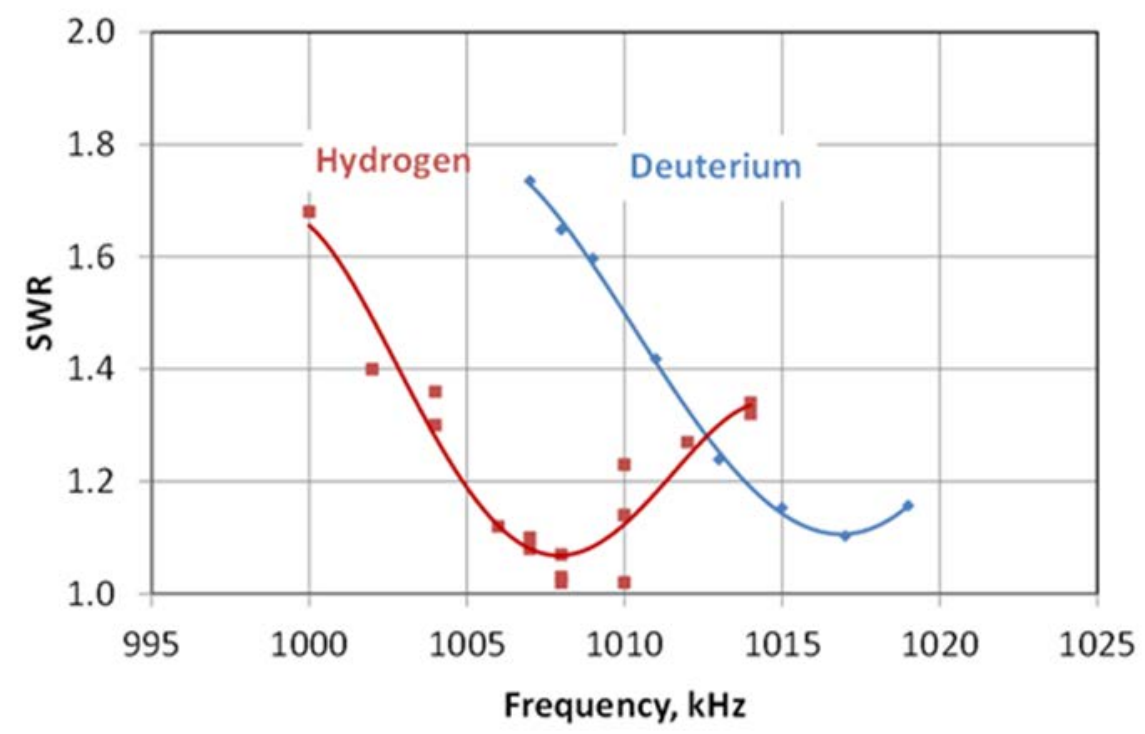

Figure 7: Difference of the matching between hydrogen and deuterium 


\subsection{Matching with different transformers and without transformer}

The matching of all oscillators operated at IPP is highly dependent on the ferrites used in the isolation transformer. This dependence is because a higher inductance of the transformer increases the $Q$ factor of the matching circuit. The standard transformer used at IPP is equipped with low $\mu$ ferrites. The inductance measured for $1 \mathrm{MHz}$ at the primary side with an open secondary side is below $10 \mu \mathrm{H}$. This transformer enables the best matching with all oscillators. In a second transformer ferrites with higher $\mu$ are installed, resulting in an inductance of $27 \mu \mathrm{H}$. With this transformer, the matching to the source is much more affected by flips of the oscillator frequency and the achievable $\cos \varphi$ is below 0.8 .

The solid state generator, however, is not affected by the different ferrites; the matching characteristic was the same regardless which of the two transformers was used.

For the matching unit of the ITER sources no isolation transformer is foreseen, because the source and the generator will be on the same potential. The matching without transformer was also tested. In this case, the 1:3 transformation of the transformer was compensated by higher values of the parallel capacitance $\mathrm{C} 1$ in Figure 1 . Figure 8 shows the SWR as a function of the series capacitance C2 with different values of C1 at a frequency of $1050 \mathrm{kHz}$. An SWR close to 1 corresponding to $50 \Omega$ at the generator output has been achieved by the use of a parallel capacitor of $17 \mathrm{nF}$ and a series capacitor of $2.25 \mathrm{nF}$.

The coupling factor, the inductance and the ohmic losses of the transformer are not exactly known as a function of the source parameters; therefore accurate calculation of the plasma load from the capacities is not possible. Without the transformer the matching circuit including the transformation by the transmission line can be calculated analytically more precisely. The impedance and reactance at the output of the amplifier has been calculated for $60 \mathrm{~kW}$ and 0.4 Pa using the measured series and parallel capacitances (see Figure 9). The series capacitance corresponds to a total inductance of $11.5 \mu \mathrm{H}$, which is reasonable for the used RF coil with six windings. The transformed plasma resistance has been calculated to 1.8 $\Omega$ from the parallel capacitance which is necessary to achieve $50 \Omega$ at the output of the amplifier or SWR=1 respectively. This value is consistent with the results achieved by transformer model calculations for inductive discharge in [12].

Because the SWR value is very sensitive to changes of the parallel capacitance $C 1$, the matching without transformer 


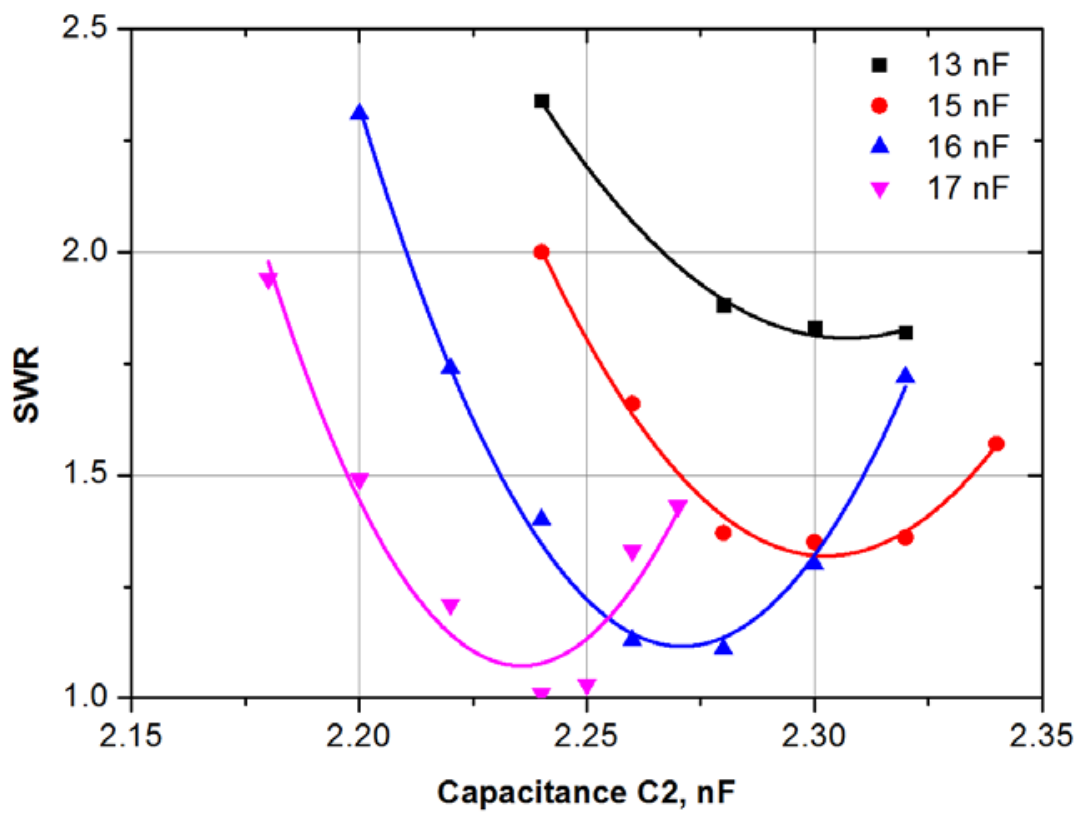

Figure 8: Matching without transformer with different values of the parallel capacitance C1

can be used in future to determine the plasma resistance as a function of the operational parameters.

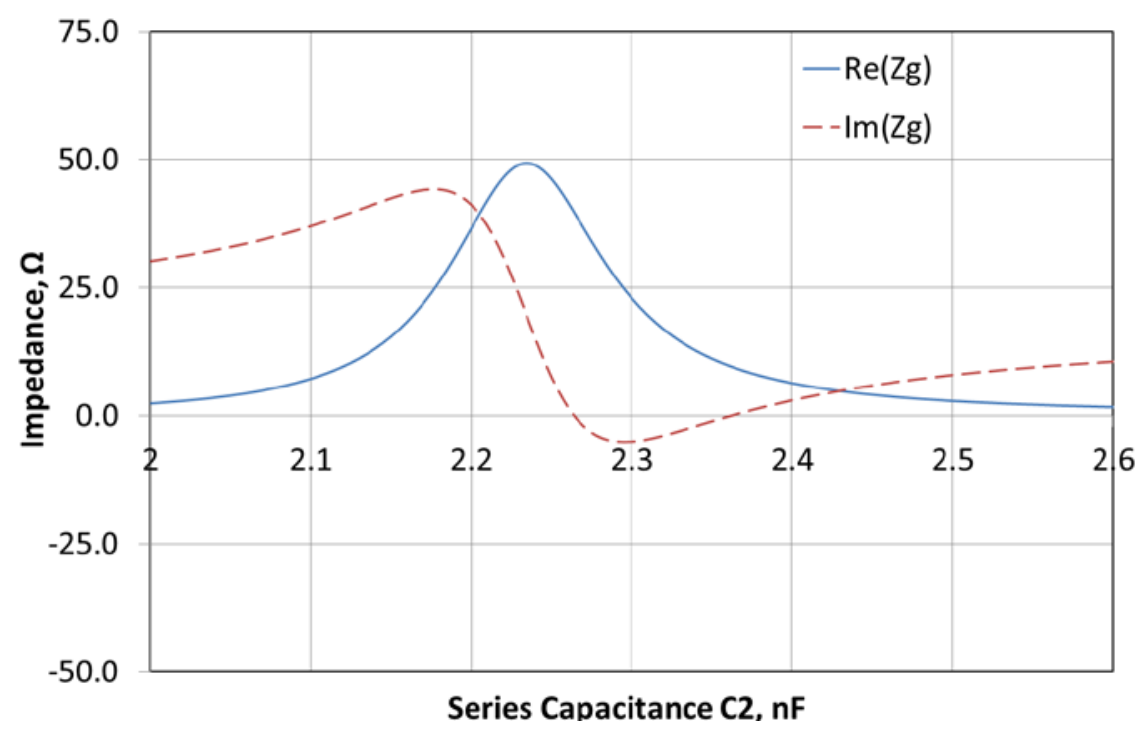

Figure 9: Impedance $\operatorname{Re}(\mathrm{Zg})$ and reactance $\operatorname{Im}(\mathrm{Zg})$ at the output of the amplifier calculated for $R=1.8$ $\Omega, L=11.5 \mu \mathrm{H}$ and $25 \mathrm{~m}$ transmission line for $1050 \mathrm{kHz}$

\subsection{Efficiency of the plasma production}

Both, the self-excited oscillator and the solid state generator, have been operated with the same matching circuit. The only way to lose power in this circuit is to heat the ferrite core transformer. This was not the case for either generator. As there are no other power losses, differences in the efficiency of plasma production can only originate from differences in the power measurement system. This has been investigated by measuring both the Ha light 
intensity along the central axis of the driver and the extracted negative ion beam current for the same source parameters operated by both generators.

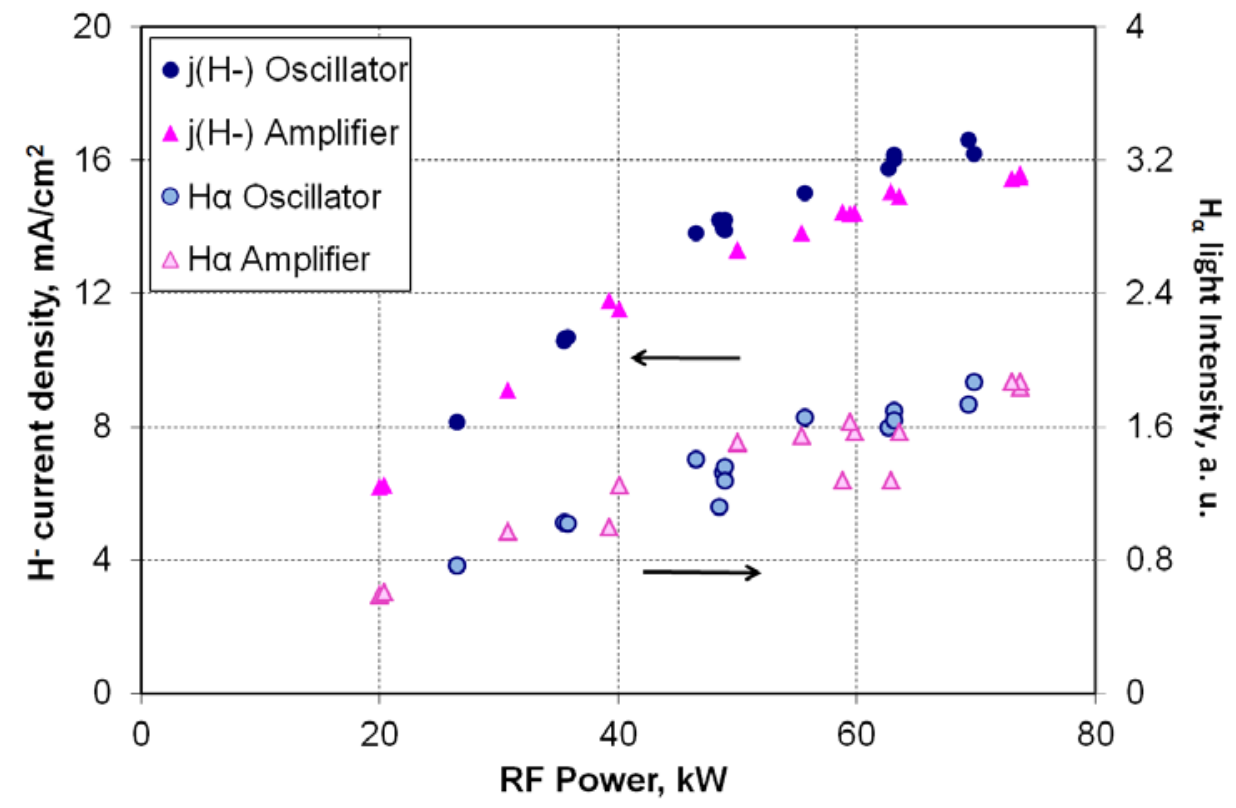

Figure 10: Power scan of the extracted $H$ current density and of the $\mathrm{H \alpha}$ light for the oscillator and the solid state generator at $0.5 \mathrm{~Pa}$

As expected, there is no clear difference of the intensity of the Ha light as the function of the power. Beam extraction in Hydrogen was carried out with the two generators on two subsequent days during the Caesium conditioning phase of the source $[9,11]$. As it is very difficult to maintain identical Caesium conditions, the maximum $\mathrm{H}^{-}$current density (see Figure 10) was slightly lower (approx. $1 \mathrm{~mA} / \mathrm{cm}^{2}$ ) with the amplifier, although the Ha light intensity was more or less the same.

\section{Summary and conclusion}

The experiments with the prototype solid state generator as a power supply for a negative Hydrogen ion source using the same matching circuit have demonstrated that matching to the plasma load is superior to that achievable with the oscillator type generator. The unsatisfactory matching of these generators results in non-negligible reflected power which represents an additional load for the transmission line and requires that the generators delivers a higher apparent power, and thus have to be more powerful. For example the power of each of the four ITER RF generators which is required for the ion source operation is $160 \mathrm{~kW}$, but the generators will have a maximum output power of $200 \mathrm{~kW}$ in order to ensure sufficient spare power.

With the prototype solid state generator perfect matching has been shown, which in contrast to the oscillator type generators, is not affected by changes to the RF circuit. Reliable operation is possible with reflected power below $1 \%$. For the case of ITER the use of 
solid state amplifiers would reduce the necessary spare power and this taken together with an efficiency of $90 \%$ would reduce the primary power from $1.4 \mathrm{MW}$ to below $0.8 \mathrm{MW}$.

Although due to the fixed frequency the matching range is smaller, it is wide enough to cover the range of all load variations caused by different source parameters in typical operation.

In these tests no reliability problems were observed, because damage to the modules of the amplifier by the reflected power has been avoided by an effective protection system.

The advantages compared to the oscillators: higher efficiency, lower costs, simplification of the system by less cooling requirements and due to the proven reliability plus the superior matching characteristics, make solid state amplifiers a very attractive option for the next generation of ion source power supplies. 


\section{References}

[1] Fantz U., Franzen P., Wünderlich D., Chemical Physics 398 (2012) 7-16; doi.org/10.1016/j.chemphys.2011.05.006

[2] Speth E., et al., Fusion Engineering and Design 46 (1999) 383-388

[3] McNeely P., Barlak M., Baldzuhn J. et al., Fusion Engineering and Design 88 (2013) 10341037

[4] Hemsworth R., et al. Nuclear Fusion, v. 49, p. 045006, 2009; doi:10.1088/0029$5515 / 49 / 4 / 045006$

[5] Rotti C., Chakraborty A.K., Bandyopadhyay M., Singh M., Gangadharan R., Ahmed I., et al., Proceedings of the 24th IAEA Fusion Energy Conference, 2012, ITR/P 5-43

[6] Kraus W., Briefi S., Fantz U., Gutmann P., Doerfler J., Review of Scientific Instruments 85, 02B309 (2014); doi.org/10.1063/1.4832137

[7] TRANSRADIO SenderSysteme Berlin AG, http://www.transradio.de

[8] E. Gaio, W. Kraus, C. Martens, R. Piovan, E. Speth, V. Toigo, Fusion Engineering and Design 82 (2007) 912-919

[9] Kraus W., Berger M., Fantz U., Franzen P., Fröschle M., Heinemann B., Riedl R., Speth E., Stäbler A., Review of Scientific Instruments 79 (2008) 02 C108

[10] Singh M., Bandyopadyay M., Bansal G., Gahlaut A., Soni J., et al., AIP Conf. Proc. 1390, 604-613(2011)

[11] P. Franzen, B. Heinemann, U. Fantz, D. Wünderlich, W. Kraus, M. Fröschle, C. Martens, R. Riedl, R. Nocentini, A. Masiello, B. Ruf, L. Schiesko, C. Wimmer, Fusion Engineering and Design 88 (2013) 3132-3140

[12] D. Sudhir, M. Bandyopadhyay, W. Kraus, A. Gahlaut, G. Bansal, and A. Chakraborty, Review of Scientific Instruments 85, 013510 (2014); doi: 10.1063/1.4863098 